Arbeitsstandards in globalen Produktionsketten

\title{
Chancen und Grenzen freiwilliger Unternehmensverantwortung
}

\author{
Unternehmensinitiativen erstellen zunehmend \\ freiwillige Verhaltenskodizes über die Arbeitsbe- \\ dingungen bei Zuliefererbetrieben. Doch diese \\ Verhaltensregeln unterscheiden sich oft in wich- \\ tigen Punkten. Zumeist ist ein Mangel an Trans- \\ parenz festzustellen, der die Überprüfung der \\ Richtlinien erschwert. \\ Von Mark Starmanns
}

V or 20 Jahren wurde die Bekleidungsindustrie erstmals mit Anti-Sweatshop-kampagnen konfrontiert. Seither versuchen viele Unternehmen, im Rahmen freiwilliger Unternehmensverantwortung die Arbeitsbedingungen in den ausgelagerten Fabriken in Niedriglohnländern zu verbessern. Dennoch berichten Medien regelmäßig über miserable Arbeitsbedingungen in solchen Fabriken. Inwiefern können freiwillige Sozialstandardinitiativen zu fairen Arbeitsbedingungen beitragen? Und wo sind die Grenzen solcher Initiativen?

Bekleidung für den deutschen Markt wird fast ausschließlich in Niedriglohnländern produziert, wo oft grundlegende Arbeits- und Menschenrechte ignoriert werden. Deshalb definieren immer mehr Bekleidungsfirmen in einem Verhaltenskodex welche Arbeitsstandards bei ihren Produzenten mindestens gelten müssen. Diese Standards entsprechen in der Regel den nationalen Arbeitsgesetzen des Produktionslandes beziehungsweise den acht Kernarbeitsnormen der Internationalen Arbeitsorganisation (1). Um dem Wildwuchs an Unternehmens-Verhaltenskodizes entgegen zu treten wurden in den letzten 20 Jahren verschiedene Sozialstandardinitiativen gegründet.

Die Unternehmensinitiativen Business Social Compliance Initiative (BSCI), Ethical Trading Initiative (ETI), Fair Labor Association (FLA), Fair Wear Foundation (FWF) nehmen Unternehmen als Mitglieder auf, wenn diese eine Gebühr zahlen und wenn sie sich dazu verpflichten, den Regeln der Initiative zu folgen (2). Hierzu gehört auch die mittelfristige Umsetzung der Arbeitsrechte in der eigenen Lieferkette. Im Gegensatz dazu hat die Initiative Social Accountability International das Fabrikzertifikat SA 8000 entwickelt. Dies setzt bei den Produzenten an und bescheinigt einer Fabrik die Einhaltung von Mindeststandards. Alle fünf Initiativen wollen dabei helfen, die Summe der Einzelbemühungen in der Standardumsetzung zu bündeln und zu koordinieren, sodass Sozialstandards effektiver und effizienter umgesetzt werden. Zusätzlich sollen Standardinitiativen den
Aktivitäten einer Firma mehr Glaubwürdigkeit verleihen. Aber in den Details unterscheiden sich die fünf erwähnten Initiativen stark voneinander. Fünf Fragen sollen helfen, die Unterschiede herauszuarbeiten und die Chancen und Grenzen privater Standardinitiativen abzustecken.

\section{Wie werden soziale Standards definiert?}

In einer freiwilligen Standardinitiative definieren Unternehmen oder verschiedene Akteure gemeinsam die komplexen Regeln der Initiative. In der Theorie verlangen Fragen nach der Glaubwürdigkeit und Legitimität einer Initiative, dass diese Entscheidungen von allen relevanten Stakeholdern getroffen werden. In Business Initiativen wie der BSCI fällen jedoch Unternehmen alleine die Entscheidungen über Standards und Regeln. In der Regel stehen Effizienzbestrebungen im Vordergrund, wodurch die Ansprüche der Arbeiter in den Hintergrund geraten.

Multi-Stakeholder-Initiativen (MSI) wird oftmals eine höhere Glaubwürdigkeit und Legitimität bescheinigt, weil verschiedene Anspruchsgruppen gemeinsam über die Standards und Regeln entscheiden. Oftmals werden MSI auch eine größere Nachhaltigkeit in der Umsetzung zugeschrieben, weil Entscheidungen im Konsens getroffen werden. Mögliche spätere Probleme werden damit frühzeitig ausdiskutiert. Doch es ist ganz entscheidend, welche Gruppe auf welche Weise in dem Prozess beteiligt ist und wen sie repräsentiert. So sind beispielsweise in keiner der vier MSI Vertreter aus den Produktionsländern oder Arbeiter direkt an den Entscheidungen beteiligt. Dies zu ermöglichen, könnte eine Aufgabe für Staaten sein.

\section{Standards für die gesamte Lieferkette?}

Die fünf Sozialstandardinitiativen sind sich einig darin, dass in ausgelagerten Fabriken mindestens Kernarbeitsnormen der Internationalen Arbeitsorganisation eingehalten werden müssen. Doch bei den Löhnen geht die Meinung weit auseinander. Zwar berufen sich alle Initiativen darauf, dass mindestens national festgelegte Mindestlöhne beziehungsweise die teilweise höheren Industrielöhne gezahlt werden müssen. Doch decken diese oftmals die grundlegenden Bedürfnisse der Arbeiter nicht ab. Regierungen und Industrieverbände fürchten bei der Festlegung zu hoher Löhne Standortnachteile. Um diesem Problem $\mathrm{zu}$ begegnen, definieren FWF und ETI in ihren Kodizes sogenannte „living wages“, die bei einer normalen 48-Stunden Arbeitswoche ein menschenwürdiges Leben sicherstellen sollten. 
Solange aber menschenwürdige Existenzlöhne nicht verbindlich von Staaten definiert sind und es zahlreiche Vorstellungen darüber gibt, wie hoch sie sein sollten, wird man in Niedriglohnländern kaum eine Fabrik finden, die hohe Existenzlöhne zahlt.

\section{Wie wird die Einhaltung der Standards kontrolliert?}

Markenfirmen und Einzelhändler führen in ihren Fabriken soziale Audits durch oder lassen die Standardinitiativen dies tun. Diese Audits sollen die aktuelle Situation in einer Fabrik festhalten. Doch Audits sind aus verschiedenen Gründen in die Kritik geraten und die ETI behauptet beispielsweise, dass Audits Geldverschwendung seien. Unter anderem wird angezweifelt, dass die verwendeten Methoden und die mangelnde Transparenz tatsächlich $\mathrm{zu}$ glaubwürdigen Ergebnissen führen. Ferner wurde nachgewiesen, dass Audits nur marginal zu Verbesserungen in den Fabriken führen (Usher/Newitt 2011; Locke et al. 2006).

Um die Glaubwürdigkeit von Audits zu verbessern, verfolgen FWF und FLA einen Verifizierungsansatz. Dabei führt jedes Mitglied in der Lieferkette Audits durch, welche dann von einem externen Audit-Team der jeweiligen Initiative stichprobenartig kontrolliert werden. Die Teams der FWF und FLA erheben den Anspruch, unabhängiger und glaubwürdiger als andere Teams zu sein, unter anderem, weil die Auditoren nicht direkt von der Fabrik bezahlt werden, die sie kontrollieren, wie es bei BSCI-Audits und beim SA 8000 Zertifikat oftmals der Fall ist (3).

Tabelle 1: Sozialstandardinitiativen

\begin{tabular}{|c|c|c|c|c|c|}
\hline & $\begin{array}{l}\text { Business Social } \\
\text { Compliance } \\
\text { Initiative (Brüssel) }\end{array}$ & $\begin{array}{l}\text { Ethical Trading } \\
\text { Initiative } \\
\text { (London) }\end{array}$ & $\begin{array}{l}\text { Fair Labor } \\
\text { Association } \\
\text { (Washington) }\end{array}$ & $\begin{array}{l}\text { Fair Wear } \\
\text { Foundation } \\
\text { (Amsterdam) }\end{array}$ & $\begin{array}{l}\text { Social } \\
\text { Accountability } 8000 \\
\text { (New York) }\end{array}$ \\
\hline $\begin{array}{l}\text { Initiativentyp, } \\
\text { Anzahl Mitglieder }\end{array}$ & $\begin{array}{l}\text { Auditieren, } \\
>700\end{array}$ & $\begin{array}{l}\text { Lernen, } \\
>70\end{array}$ & $\begin{array}{l}\text { Verifizierung, } \\
>70\end{array}$ & $\begin{array}{l}\text { Verifizierung, } \\
>70\end{array}$ & $\begin{array}{l}\text { Zertifizierung, } \\
>2000 \text { zertifizierte } \\
\text { Fabriken }\end{array}$ \\
\hline 1. Wer definiert die Regeln? & Unternehmen & $\begin{array}{l}\text { Unternehmen, NGOs, } \\
\text { Gewerkschaften }\end{array}$ & $\begin{array}{l}\text { Unternehmen, NGOs, } \\
\text { Universitäten }\end{array}$ & $\begin{array}{l}\text { Unternehmensverbände, } \\
\text { NGOs, Gewerkschaften }\end{array}$ & $\begin{array}{l}\text { Unternehmen, } \\
\text { Berater }\end{array}$ \\
\hline $\begin{array}{l}\text { 2. Welche Standards } \\
\text { werden definiert? }\end{array}$ & $\begin{array}{l}\text { ILO Kern- } \\
\text { arbeitsnormen; } \\
\text { Mindestlöhne }\end{array}$ & $\begin{array}{l}\text { ILO Kernarbeits- } \\
\text { normen; Living Wages } \\
\text { (Berechnung in- } \\
\text { transparent) }\end{array}$ & $\begin{array}{l}\text { ILO Kernarbeitsnormen; } \\
\text { Mindestlöhne } \\
\text { (“Fair Wages" Projekt) }\end{array}$ & $\begin{array}{l}\text { ILO Kernarbeitsnormen; } \\
\mathrm{BSCl}, \mathrm{ETI}, \mathrm{FLA}, \mathrm{FWF}\end{array}$ & $\begin{array}{l}\text { ILO Kernarbeits- } \\
\text { normen; } \\
\text { Living Wages } \\
\text { (Berechnung } \\
\text { intransparent) }\end{array}$ \\
\hline $\begin{array}{l}\text { 3. Wie wird Einhaltung der } \\
\text { Standards kontrolliert? }\end{array}$ & $\begin{array}{l}\text { Audits durch SAAS-ak- } \\
\text { kreditierte Auditoren } \\
\text { (66\% der Lieferanten } \\
\text { aus Risiko-Ländern in } \\
\text { 3,5 Jahren); i.d.R. zahlt } \\
\text { Fabrik; einzelne „Sur- } \\
\text { veillance Audits“ }\end{array}$ & $\begin{array}{l}\text { keine Auditpflicht, viele } \\
\text { Firmen aber auditieren } \\
\text { und zahlen selbst; } \\
\text { NGOs und Gewerk- } \\
\text { schaften bewerten } \\
\text { Jahresberichte der } \\
\text { Unternehmen }\end{array}$ & $\begin{array}{l}\text { Mitglied auditiert } \\
\text { selbst \& zahlt selbst; } \\
\text { Verifizierung durch } \\
\text { FLA akkreditierte } \\
\text { Auditoren (FLA zahlt, } \\
\text { ca. } 4 \% \text { jährlich) }\end{array}$ & $\begin{array}{l}\text { Mitglied auditiert in } 3 \text { Jah- } \\
\text { ren } 90-100 \% \text { der Lieferan- } \\
\text { ten und zahlt selbst; } \\
\text { Verifizierung durch lokale } \\
\text { FWF Audit-Teams (FWF } \\
\text { zahlt, } 10 \% \text { in } 3 \text { Jahren) } \\
\text { jährlich Work Plan \& Ma- } \\
\text { nagement System Audits }\end{array}$ & $\begin{array}{l}\text { Zertifizierung durch } \\
\text { SAAS akkreditierte Au- } \\
\text { ditoren (Fabrik zahlt); } \\
\text { Zertifikat drei Jahre } \\
\text { gültig; "Surveillance- } \\
\text { Audit" nach sechs } \\
\text { Monaten }\end{array}$ \\
\hline $\begin{array}{l}\text { 4. Unterstützung der } \\
\text { Lieferanten }\end{array}$ & Workshops & $\begin{array}{l}\text { ETI Principles of Imple- } \\
\text { mentation }\end{array}$ & $\begin{array}{l}10 \text { Principles of Fair } \\
\text { Labor \& Responsible } \\
\text { Sourcing }\end{array}$ & $\begin{array}{l}\text { jährlich Social Report der } \\
\text { Mitglieder \& Zusammen- } \\
\text { fassung der Management }\end{array}$ & Workshops \\
\hline 5. Transparenz & $\begin{array}{l}\text { keine Pflicht-Berichte } \\
\text { auf Mitgliederbasis }\end{array}$ & $\begin{array}{l}\text { Jahresberichte der Mit- } \\
\text { glieder nur ETI zugäng- } \\
\text { lich }\end{array}$ & $\begin{array}{l}\text { Tracking Charts; einige } \\
\text { Firmen veröffentlichen } \\
\text { Fabriklisten }\end{array}$ & System Audits & $\begin{array}{l}\text { Liste zertifizierter } \\
\text { Fabriken }\end{array}$ \\
\hline
\end{tabular}

Quelle: Eigene Darstellung
Arbeitsrechtsvertreter schlagen vor, dass die Arbeiter im Zentrum von Verbesserungsprozessen stehen und sich aktiv einbringen können, was aber selten geschehe. Um Arbeitern dies zu ermöglichen, wird versucht, zivilgesellschaftliche oder staatliche Akteure aus der Region stärker zu integrieren.

\section{Wie werden die Lieferanten bei der Umset- zung unterstützt?}

Arbeitsbedingungen verbessern sich nicht alleine dadurch, dass Arbeitsstandards definiert und auditiert werden (siehe beispielsweise Nadvi et al. 2011; Locke et al. 2009). Sowohl die Standardinitiative als auch die beteiligten Unternehmen müssen sich aktiv für die Verbesserung der Arbeitsbedingungen engagieren. Dies geschieht bisher in sehr unterschiedlichem Ausmaß und es existieren nur wenige wissenschaftliche Evaluierungen dazu.

Die Umsetzung von Sozialstandards wird zu einer besonders großen Herausforderung, wenn der Produzent die sozialen Anforderungen vor dem Hintergrund der geforderten Qualität, Stückzahl, Lieferzeit und dem vereinbarten Preis nicht umsetzen kann. So fordern Einkäufer oftmals für die nächste Saison einen um zehn bis 20 Prozent niedrigeren Preis für das gleiche Produkt, obwohl die Produktionskosten im Land des Zulieferers steigen. Hier setzen sowohl die FWF als auch die ETI mit konkreten Maßnahmen an, um die Einkaufsbedingungen so anzupassen, dass sie die Einführung von Standards nicht verhindern. 


\section{"Staatliche Regulierung kann Kooperation für bessere Arbeitsbedingungen erleichtern."}

Um den Arbeitern und anderen Gruppen besser zu ermöglichen, sich über die Verletzung ihrer Rechte zu beschweren, haben die FWF und FLA eigene Beschwerdemechanismen eingerichtet. Diese sollen den Arbeitern einen sicheren Zugang zu einer unabhängigen Stelle vor Ort ermöglichen, die auch ihr Vertrauen genießt. Der Mechanismus reduziert die Relevanz der Audits und befähigt die Arbeiter, für sich selbst zu sprechen. Jedoch konnte bisher alleine die Existenz eines solchen Mechanismus die Arbeiter nicht hinreichend motivieren, ihre Rechte auch einzufordern. Hier könnten Staaten unterstützend eingreifen.

\section{Wie erfolgreich sind die Initiativen?}

Die Wirksamkeit privater Sozialstandardinitiativen im Bekleidungssektor ist wenig erforscht. Etwas ältere Studien bestätigen eher die Kritik zivilgesellschaftlicher Gruppen und argumentieren, dass die Haupteffekte in den Bereichen Arbeitssicherheit und Gesundheit erzielt würden, wohingegen sich eher versteckte Rechte wie die Gewährung von Gewerkschaftsfreiheit oder Frauendiskriminierungen kaum verbesserten (Institute of Development Studies 2006). Natürlich schreiben in den Jahresberichten alle Initiativen Positives über die eigene Wirkung und zeigen ihren Impact anhand sozialer Audits auf. Doch aufgrund der Kritik an sozialen Audits sind solche Fortschrittsberichte eher fragwürdig.

Eine höhere Transparenz der Initiativen zu den Produktionsstätten und zum eigenen sozialen Fußabdruck insbesondere gegenüber lokalen Akteuren würde Kooperationen zwischen Unternehmen und Arbeitervertretern vereinfachen. Doch freiwillige Maßnahmen konnten die Initiativen bisher nicht dazu bewegen, diese Transparenz zu erbringen, auch wenn einzelne Unternehmen wie Patagonia, Switcher oder honestby zeigen, dass es möglich ist, transparent und erfolgreich zu sein. Staatlich verbindliche Regeln zur Transparenz, wie sie die Europäische Kommission anvisiert, könnte ein „level playing field“ zwischen Unternehmen herstellen.

\section{Fazit}

Alle hier analysierten Sozialstandardinitiativen verlangen von ihren Mitgliedern beziehungsweise den Betrieben, die sie zertifizieren, sich mehr oder weniger intensiv in einen Verbesse- rungsprozess einzubringen. Dabei berücksichtigen Multi-Stakeholder-Initiativen aufgrund ihrer Governance-Struktur naturgemäß die Arbeiter stärker als reine Business-Initiativen. Im Vergleich zwischen der BSCI und der FWF hat dies dazu geführt, dass die BSCI einen eher Management-orientierten Ansatz umsetzt, die FWF dagegen einen eher Arbeiter-orientierten Ansatz. So fordert die FWF im Gegensatz zur BSCI von ihren Mitgliedern die Zahlung von „living wages“. Weil aber viele FWF-Mitglieder ihre Fabriken mit Einkäufern teilen, denen die Zahlung von Mindestlöhnen ausreicht, haben Mitglieder der FWF erhebliche Schwierigkeiten, Existenzlöhne überhaupt umzusetzen. Auch hier könnte staatliche Regulierung versuchen, ein „level playing field“ zu fördern, das Kooperation für bessere Arbeitsbedingungen erleichtert.

\section{Anmerkungen}

(1) Im Internet unter: www.ilo.org

(2) Business Social Compliance Initiative (BSCl), Ethical Trading Initiative (ETI), Fair Labor Association (FLA), Fair Wear Foundation (FWF), Social Accountability International (SAI) sind die bisher für deutsche Unternehmen wichtigsten Initiativen. Die Auflistung ist nicht umfassend.

(3) Dabei spricht für das SA 8000 Zertifikat, dass jede zertifizierte Fabrik öffentlich gelistet ist, was theoretisch Kritik ermöglicht.

\section{Literatur}

Institute of Development Studies: Report on the ETI Impact Assessment: The ETI code of labour practice: do workers really benefit? Sussex 2006. Locke, R. / Amengual, M. / Mangla, A.: Virtue out of Necessity? Compliance, Commitment and the Improvement of Labor Conditions in Global Supply Chains. In: Politics \& Society 37, 3/2009, S. 319-351.

Locke, R. / Qin, F. / Brause, A.: Does Monitoring Improve Labor Standards? Lessons from Nike. In: Industrial and Labor Relations Review 61, 1/2006, S. 3-31.

Nadvi, K. / Lund-Thomsen, P. / Xue, H. / Khara, N.: Playing against China: global value chains and labour standards in the international sports goods industry. In: Global Networks 11, 3/2011, S. 334-354.

Usher, A. / Newitt, K.: Beyond auditing. Tapping the full potential of labour standards promotion. IDS. Utrecht 2011.

\section{AUTOR + KONTAKT}

Mark Starmanns arbeitet an der Universität Zürich und ist Mitgründer des Netzwerks Faire Mode. Das Netzwerk hilft Konsumenten, öko-faire Mode zu finden, und berät Firmen, die nachhaltiger produzieren wollen.

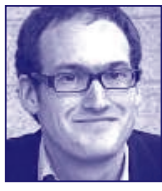

Universität Zürich, Institut für Geographie, Winterthurer Str. 190, 8057 Zürich. Tel.: +41 44 6355209, Fax: +41 44 6356848, E-Mail: mark.starmanns@geo.uzh.ch, Internet: www.geo.uzh.ch 
Copyright (C) 2012, IÖW und oekom Verlag. Die Nutzung des Artikels ist Abonnenten von Ökologisches Wirtschaften vorbehalten. Nachdruck und Vervielfältigung des Artikels einschließlich Speicherung und Nutzung auf optischen und elektronischen Datenträgern nur mit Zustimmung der Redaktion von Ökologisches Wirtschaften (http://www.oekologischeswirtschaften.de). 